\title{
Sleep Hygiene and Recovery Strategies in Elite Soccer Players
}

\section{Mathieu Nédélec, Shona Halson,}

\section{Barthélémy Delecroix, Abd-Elbasset}

\section{Abaidia, Said Ahmaidi \& Gregory}

\section{Dupont}

\section{Sports Medicine}

ISSN 0112-1642

Sports Med

DOI 10.1007/s40279-015-0377-9
2015, Vol. 45, No. 9 (PI ISSN: 0112-1642 (Print); $117 \mathrm{~s}$

ONLINE FIRST

Sports Medicine

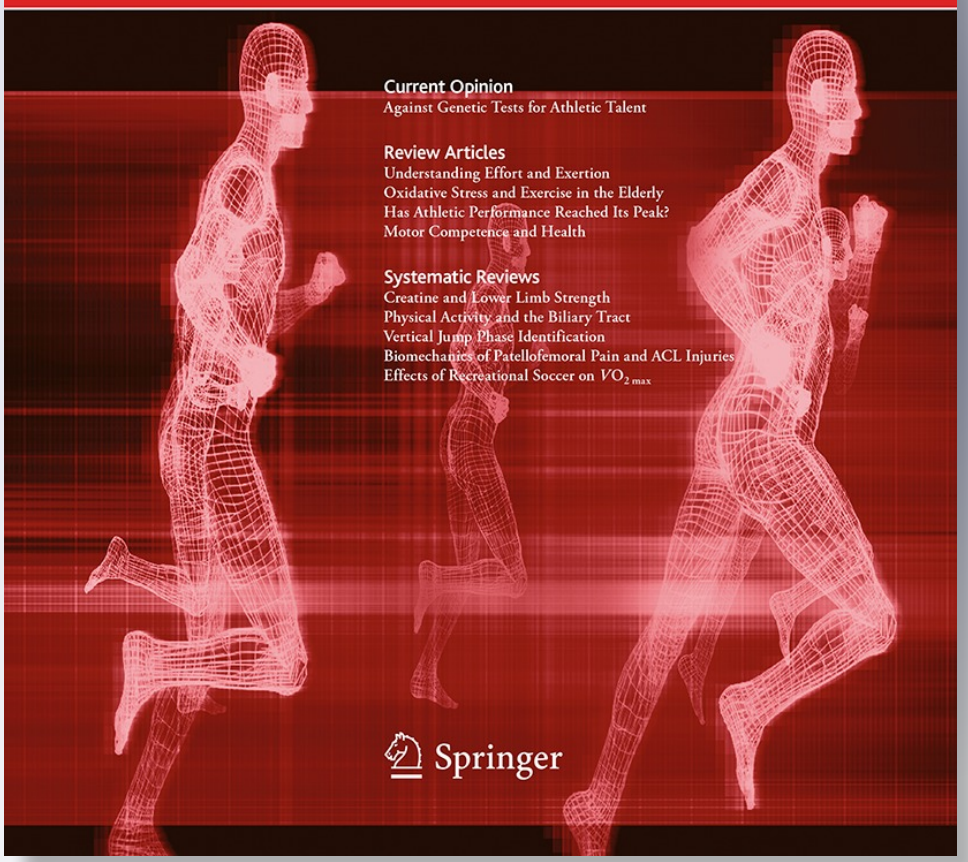

包 Springer 
Your article is protected by copyright and all rights are held exclusively by Springer International Publishing Switzerland. This eoffprint is for personal use only and shall not be self-archived in electronic repositories. If you wish to self-archive your article, please use the accepted manuscript version for posting on your own website. You may further deposit the accepted manuscript version in any repository, provided it is only made publicly available 12 months after official publication or later and provided acknowledgement is given to the original source of publication and a link is inserted to the published article on Springer's website. The link must be accompanied by the following text: "The final publication is available at link.springer.com". 


\title{
Sleep Hygiene and Recovery Strategies in Elite Soccer Players
}

\author{
Mathieu Nédélec $^{1,2} \cdot$ Shona Halson $^{3} \cdot$ Barthélémy Delecroix $^{2,4} \cdot$ Abd-Elbasset Abaidia $^{2,4}$. \\ Said Ahmaidi ${ }^{1} \cdot$ Gregory Dupont $^{2,4}$
}

(C) Springer International Publishing Switzerland 2015

\begin{abstract}
In elite soccer, players are frequently exposed to various situations and conditions that can interfere with sleep (e.g., playing night matches interspersed with 3 days; performing activities demanding high levels of concentration close to bedtime; use of products containing caffeine or alcohol in the period preceding bedtime; regular daytime napping throughout the week; variable wake-up times or bedtime), potentially leading to sleep deprivation. We outline simple, practical, and pharmaceutical-free sleep strategies that are coordinated to the constraints of elite soccer in order to promote sleep. Sleep deprivation is best alleviated by sleep extension; however, sleep hygiene strategies (i.e., consistent sleep pattern, appropriate napping, and active daytime behaviors) can be utilized to promote restorative sleep. Light has a profound impact on sleep, and sleep hygiene strategies that support the natural environmental light-dark cycle (i.e., red-light treatment prior to sleep, dawn-simulation therapy prior to waking) and prevent cycle disruption (i.e., filtering short wavelengths prior to sleep) may be beneficial to elite soccer players. Under conditions of inordinate stress, techniques such as brainwave entrainment and meditation are promising sleep-promoting strategies, but future studies are required to ascertain the applicability of these techniques to
\end{abstract}

Mathieu Nédélec

matnedelec@orange.fr

1 Université de Picardie Jules Verne, UFR STAPS, Allée Paschal Grousset, 80025 Amiens Cedex 1, France

2 LOSC Lille Métropole Football Club, Camphin-en-Pévèle, France

3 Department of Physiology, Australian Institute of Sport, Belconnen, Canberra, ACT, Australia

4 Université Lille Nord de France, Lille, France elite soccer players. Consuming high-electrolyte fluids such as milk, high-glycemic index carbohydrates, some forms of protein immediately prior to sleep, as well as tart cherry juice concentrate and tryptophan may promote rehydration, substrate stores replenishment, muscle-damage repair and/or restorative sleep. The influence of cold water immersion performed close to bedtime on subsequent sleep is still debated. Conversely, the potential detrimental effects of sleeping medication must be recognized. Sleep initiation is influenced by numerous factors, reinforcing the need for future research to identify such factors. Efficient and individualized sleep hygiene strategies may consequently be proposed.

\section{Key Points}

Although sleep provides psychological and physiological functions that are considered critical to optimal recovery, various situations and conditions in elite soccer-including playing night matches with late kick-off, light, congested scheduling, and travel-can interfere with sleep.

Sleep hygiene strategies can be utilized during off days to promote restorative sleep, whereas sleep hygiene strategies related to light and nutrition can be used before and/or during the night after night matches.

\section{Introduction}

Soccer involves many physically demanding activities, including sprinting, changes in running speed, changes of direction, jumps and tackles, as well as technical actions 
such as dribbling, passing, and shooting. These activities lead to post-match fatigue that is mainly linked to a combination of glycogen depletion, muscle damage, and mental fatigue [1]. In elite soccer, recovery strategies are required during congested schedules to alleviate post-match fatigue, regain performance levels, and reduce the risk of injury [1]. Sleep provides a number of important psychological and physiological functions that may be fundamental to the recovery process. During sleep, which includes five distinct stages (stages 1, 2, 3, 4, and rapid-eye-movement stage), metabolic activity is at its lowest point (i.e., slow breathing, low heart rate, and low cerebral blood flow) and the endocrine system increases the secretion of growth hormone via the pituitary gland, allowing physiological restitution $[2,3]$. Learning and motor memory are associated with slow-wave sleep, rapid-eye-movement sleep [46], and sleep spindles [7] during the night. Consequently, sleep may assist in the recovery of the nervous and metabolic cost imposed by the waking state [8].

Most studies have examined the negative effects of sleep deprivation on aspects of performance, with research investigating the effect of improved sleep being rare [912]. Maximizing sleep may be one method of enhancing performance that has gained little scientific attention. To our knowledge, only Mah et al. $[13,14]$ have examined the effects of sleep extension on subsequent performance. Collegiate basketball players were instructed to obtain as much nocturnal sleep as possible during an experimental period of 5-7 weeks, with a minimum goal of $10 \mathrm{~h}$ in bed each night. Objective sleep time effectively increased during the sleep extension period compared with baseline by $110.9 \pm 79.7 \mathrm{~min}$. Results showed a faster timed sprint following sleep extension $(16.2 \pm 0.61$ vs. $15.5 \pm 0.54 \mathrm{~s}$, $p<0.001)$; an improvement in shooting accuracy by $\sim 9 \%$; a faster reaction time $(310.8 \pm 77.1$ vs. $274.5 \pm 42.0 \mathrm{~ms}, \quad p<0.01) ;$ and subjective increased physical recovery [14]. Increasing the sleep time of five swimmers from their usual sleep/wake patterns (not stated) to $10 \mathrm{~h}$ per night for 6-7 weeks led to improvements in $15 \mathrm{~m}$ sprint, reaction time, and turn time following the sleep extension period [13]. However, these studies present some limitations. Athletes were likely facing a sleep debt at the start of the study, and the positive effects of sleep extension may differ when athletes with no sleep debt are studied. However, sub-optimal sleeping patterns are frequently noted in athletic populations [15], reinforcing the validity of present studies. As no control group was present, it should not be excluded that the collegiate athletes (aged 18-22 years) followed throughout the sleep extension period likely developed their technical skills (i.e. free throw and 3-point shooting accuracy; turn time; kick strokes) as a result of the training rather than sleep extension.
A common issue for recovery from training or competition relates to sub-optimal sleeping patterns noted in athletic populations [15]. While athletes may obtain a similar amount of sleep as healthy control subjects (total sleep time between 7 and $8 \mathrm{~h}$ ) $[15,16]$ over a period of several days, significant differences between controls and athletes for other sleep parameters (e.g. sleep latency, time awake, sleep efficiency) suggest the quality of athlete sleep is inferior [15]. Sleep impairment may be acutely dictated by the constraints of elite soccer (e.g. playing night matches with late kick-off influenced by television networks; congested scheduling; travel) $[17,18]$ and/or may be chronically self-imposed (i.e., poor sleep hygiene). In order to support the present review article content, we performed in February 2015 a survey about the sleep complaints of 19 professional soccer players after night matches (start times ranging from 8:00 to 9:00 pm) in a French top-level team participating in the UEFA (Union of European Football Associations) Europa League (unpublished observations). A total of $95 \%(N=18)$ of players indicated worse sleep in the nights after night matches. A case study revealed that a top-level European soccer player followed over a 21-day period slept for significantly less time $(5.2 \mathrm{~h})$ following night matches (kick off after 6:00 pm) than after training days $(8.8 \mathrm{~h}$ ) and day matches (kick off 3:30-6:00 pm; $8.3 \mathrm{~h}$ ) [17]. The constraints of elite soccer are related to inadequate sleep hygiene, e.g., scheduling high-intensity exercise and/or performing activities demanding high levels of concentration close to bedtime; use of products containing caffeine or alcohol in the period preceding bedtime; daytime napping regularly throughout the week; variable wake-up times or bedtime [19]. Consequently, knowledge on the complex effects of sleep disorders among elite soccer players needs to be supplemented with sufficient understanding of sleep's role in recovery, and possible sleep hygiene strategies to alleviate these issues [20].

The term 'sleep hygiene' was first used by Hauri [21] in the context of providing recommendations for patients to help them improve their insomnia. Primary sleep hygiene strategies originally addressed caffeine, alcohol, and exercise; they were derived from scientific studies and/or clinical observations of patients with poor sleep [22]. Scientific studies were conducted to confirm the potential detrimental effects of caffeine [23] or alcohol [24] on sleep and/or the potential positive effects of exercise on sleep [25]. Strategies have subsequently evolved with increased understanding of other behavioral factors that improve or worsen sleep (e.g. cognitive features, sleep environment). Sleep hygiene strategies currently refer to a list of behaviors, environmental conditions, and other sleep-related factors that are believed to promote improved quantity and quality of sleep (for a review, see Stepanski and Wyatt 
[22]). These recommendations are generally aimed at having the individual avoid behavior that interferes with a normal sleep pattern and/or engage in behavior that promotes good sleep. The fundamental difference between recovery interventions with established protocols (e.g., cold water immersion, compression garments, electrical stimulation) [26] and sleeping lies in the fact that sleep initiation does not depend entirely on the willingness of the athlete. Sleep/wake control circuits function like a flip-flop switch that is stable in either of its extreme positions (asleep or awake); once a state is established, it tends to be self-sustaining and to resist transition to the opposing state [27]. However, in transitional periods (i.e., falling asleep or waking up), when both wake-inducing and sleep-inducing neural systems are partially active at the same time, the flip-flop switch is unstable; whichever system is more active soon becomes fully engaged and shuts off the opposing system, establishing a clear state of sleep or wakefulness [27]. Consequently, simple, practical, and pharmaceutical-free sleep hygiene strategies that players might self-administer acutely while attempting to fall asleep or just prior to sleep are required to help them 'activate the switch', i.e., falling asleep [27].

The present review article outlines possible sleep hygiene strategies that are coordinated to the constraints of competition-using soccer (Association Football) as the main sport of interest-in order to promote sleep. Sleep hygiene strategies reviewed address (1) sleep hygiene strategies during off days; (2) light, relaxation, brainwave entrainment, and sleeping medication; and (3) nutrition and cold water immersion. A computerized literature search was performed using PubMed between September 2014 and June 2015 with the following keywords used in different combinations: 'sleep', 'sleep loss', 'sleep restriction' 'sleep deprivation', 'sleep extension', 'recovery', 'fatigue', 'glycogen', 'inflammation', 'immunity', 'injury', 'soccer', 'football', 'match', 'training', 'stress', 'relaxation' 'travel', 'light', 'strategy', 'nap', 'nutrition', 'diet', 'hydration', 'temperature', 'caffeine', 'alcohol', 'screen', 'insomnia', and 'hygiene'. All titles and abstracts were carefully read, and relevant articles were retrieved for review. In addition, the reference lists from both original and review articles were also reviewed. The negative effects of travel across multiple time zones on sleep [28] and related sleep hygiene strategies are not addressed in the present review as travel of this nature is limited in European domestic leagues or UEFA Champions League. Additionally, it is not the aim of the present review to assess the effects and related sleep hygiene strategies of terrestrial altitude on sleep [29], Ramadan [30], disturbed sleep and nightmares before competition [31], or sleep apnea [32].

\section{Sleep Hygiene Strategies During Off Days}

Sleep curtailment encountered by players during training and competitive days may lead to large amounts of additional sleep on off days when the number of commitments that compete for the players' time is reduced [33]. Players anecdotally report extended sleep periods (i.e., sleeping late in the morning, long nap) when there are fewer morning requirements. However, a set of sleep hygiene strategies should be followed during off days in order to promote restorative sleep. First, developing and following a regular bedtime and wake time routine is a fundamental sleep hygiene strategy [21]. Adherence to regular bedtime and wake time promotes optimal sleep propensity and consolidation due to sleeping in the phase range of circadian promotion of sleep, and proper, stable phase alignment of the circadian timekeeping system due to regularly timed exposure to environmental and indoor light [22]. A sleep time difference of more than $1 \mathrm{~h}$ between training days and days off-particularly evident among evening chronotypes - is considered a sign of 'social jet lag' [34]. Excessively long times in bed seem related to fragmented and shallow sleep, and is one of the important factors that perpetuates insomnia $[21,35]$. It has been shown that an 8 -week marked restriction of time available for sleep improves total sleep time and sleep efficiency, and is consequently an effective treatment for insomnia [35]. In this respect, players should avoid sleeping too late in the morning off days. Second, daytime nap timing and duration should be carefully chosen. Daytime napping is encouraged as a strategy to extend sleep; napping behavior for team sport athletes was documented by Lastella et al. [36]. Nap frequency (i.e., the percentage of days on which a nap is taken) was $11 \%$ among team sport athletes, with a mean duration of 00:59 \pm 01:02 h:min [36], which may be too long. Brief naps (i.e., 5-30 min) improve performance, whereas longer naps ( $>30 \mathrm{~min}$ ) are associated with awakening from deep non-rapid-eye-movement sleep, a loss of productivity and sleep inertia [37-39]. However, the benefits of brief naps over longer naps may depend on the preceding wakefulness period [39]. Regarding the nap timing, sleep efficiency is better, sleep latency is shorter, and the amount of slow-wave sleep is greater with a nap taken closer to the afternoon circadian dip in alertness than with a nap taken during the morning or evening (around 8:00-10:00 pm) forbidden zone for sleep [40, 41]. Third, as chronic partial sleep loss is the most important cause of increased daytime sleep tendency [33], players may be tempted by minimal daytime behaviors during off days (e.g., staying on sofa exposed to indoor lighting) rather than active behaviors. However, neurometabolic theories of sleep function propose that sleep repays the neural and 
metabolic cost of waking [8]. Consequently, players may be encouraged to be gently active throughout the day in order to sleep continuously through the subsequent night [33]. Getting up and spending $10 \mathrm{~h}$ only out of bed (i.e., $10 \mathrm{~h}$ of wakefulness) may not provide enough waking sleep deprivation to foster a continuous occurrence of the constitutional nightly requirement [33]. In this respect, daytime behaviors such as light exercise (e.g., brisk walking) [42] and bright-morning light exposure [43] may be recommended as behaviors promoting restorative night sleep without inducing extra fatigue and/or muscle soreness.

\section{Sleep Hygiene Strategies}

\subsection{Light}

Elite soccer players are exposed to bright light in the stadium and also after the match (e.g., light in the airport, light in the plane, and light from the screens of electronic floodlit media devices), which may have an impact on sleep. In the survey on the sleep habits after night matches, $79 \%(N=15)$ of players reported using technology (i.e., television viewing, computer/laptop, smart phones) before going to bed (unpublished observations). Light may suppress melatonin and may therefore influence sleep [44-46]. Melatonin ( $N$-acetyl-5-methoxytryptamine) is derived from tryptophan and is mainly produced by the pineal gland [47]; it plays an important role in transmission of time information from the body clock to the peripheral oscillators located in most human cells [28]. Studies that assessed the relationship between the wavelength of light and its negative effect on sleep (i.e., alerting response) reported superiority of short wavelength light (e.g., blue $470 \mathrm{~nm}$, and green $525 \mathrm{~nm}$ ) with attenuated evening increase in melatonin, melatonin rhythm shift, and attenuated decrease in core body temperature and heart rate [44, 48, 49]. Conversely, red light $(660 \mathrm{~nm})$ has no effect on melatonin suppression and slightly shortens the time before dim-light onset of melatonin secretion [48]. The present section reviews sleep hygiene strategies to best utilize light in order to support rather than disrupt the natural environmental light-dark cycle before and during night sleep.

\subsubsection{Evening Light Exposure}

During a night soccer match, soccer players are required to perform at their peak at a time that is incongruent to their circadian rhythm. Circadian rhythm sleep disorders result from a misalignment between the timing of the circadian rhythm and the external environment (e.g., shift work) [50]. Consequently, soccer players may be in some way compared to night shift workers. Night soccer match start times may range from 8:00 pm (e.g., French Ligue de Football Professionnel) to 10:00 pm (e.g., Spanish Liga de Fútbol Profesional), including $8: 45 \mathrm{pm}$ for the UEFA Champions League. Rahman et al. [51] examined the effects of glasses fitted with short-wavelength filters $(0 \%$ transmission $<480 \mathrm{~nm}$ ) during night shifts on sleep and performance in nurses. Results showed that under filtered light intervention (blue blockers), total sleep time increased (40 $\mathrm{min}$ ), wake after sleep onset was reduced $(22 \mathrm{~min}$ ), sleep-onset latency was reduced ( $8 \mathrm{~min}$ ), sleep efficiency was increased, and salivary melatonin levels were significantly higher during the night following intervention compared with baseline after night shifts without using optical filters. Reaction time and throughput on vigilance tests were similar to daytime performance under filtered light intervention. The present results suggest that the use of glasses with short-wavelength filters $(0 \%$ transmission $<480 \mathrm{~nm}$ ) after the match may improve nighttime sleep during the night following intervention. Filtering short wavelengths during early morning (e.g., in the airport, travelling from airport) may consequently allow a smoother transition to the morning sleep period among soccer players.

Light may be used immediately before sleeping to promote sleep. Zhao et al. [52] determined the effect of red light on the sleep quality and serum melatonin level of female Chinese basketball players. The participants exposed to red-light treatment received $30 \mathrm{~min}$ of total body irradiation from a red-light therapy instrument (average wavelength: $658 \mathrm{~nm}$; light dose: $30 \mathrm{~J} / \mathrm{cm}^{2}$ ) every night for 14 days. The 14-day whole-body irradiation with red-light treatment improved the players' subjective sleep quality measured by the Pittsburgh Sleep Quality Index [53]. Additionally, only players in the red-light treatment group demonstrated improvements in serum melatonin level (post-intervention: $38.8 \pm 6.7 \mathrm{pg} / \mathrm{ml}$ vs. pre-intervention: $22.2 \pm 7.2 \mathrm{pg} / \mathrm{ml} ; p<0.01)$. Consequently, redlight treatment is a possible nonpharmacologic and noninvasive therapy to prevent sleep disruption after a night soccer match. However, future studies are required to assess whether red-light therapy effectively improves objective sleep measurements. Additionally, using glasses with short-wavelength filters is a sleep hygiene strategy that is easier to use after a match for a soccer team than a cumbersome red-light therapy instrument.

\subsubsection{Morning Light Exposure}

Soccer players may benefit from receiving light therapy prior to waking. Thompson et al. [46] examined the effects of a single simulated dawn during the last $30 \mathrm{~min}$ of an 8-h night sleep on the subsequent dissipation of sleep inertia. Dawn simulation involves the gradual increase in 
illuminance of low-intensity light (i.e., starting at 0.001 lux and rising to 300 lux following a sigmoidal illumination ramp) prior to waking from sleep [46]. Sleep inertia is a transient state between sleep and feeling fully awake that can persist for up to $2 \mathrm{~h}$ post-waking [54]; the duration and severity of sleep inertia depend on the preceding sleep length, the presence of prior sleep deprivation, and individual chronotype [46]. Changes in simulated work and physical performance were obtained among eight active subjects showing a late chronotype [55] and reporting difficulty with morning waking ( $\geq 45 \mathrm{~min}$ to feel fully awake [56]). Results showed that waking in the dawnsimulation condition increases subjective alertness and ratings of perceived sleep quality and improve both cognitive (i.e., problem solving and reaction time) and physical performance $(4.7 \%$ performance improvement in a 4-km cycling time trial, $p=0.07$ ) after waking compared with control (i.e., complete darkness). The reduction in melatonin-induced soporific [57] effects and/or the greater increase in intestinal temperature post-waking in the dawnsimulation condition may explain these results. Future studies are required to extrapolate these results to professional athletes as well as to early chronotypes. As some teams may occasionally lightly train the day after a match and especially during a congested schedule, soccer players-and especially late chronotypes-who encounter sleep disruption after a match played at night but are still required to attend recovery sessions the following morning may benefit from acute dawn-simulation therapy prior to waking [58]. It appears to be a simple and practical sleep hygiene strategy that players might self-administer acutely. As a predominant issue with dawn-simulation research is measuring the quantity of light that penetrates through the eyelid, a compromise between benefiting from dawn-simulation therapy and benefiting from a dark environment by using eye shades [59] is required.

Finally, another potential source of light is the light emanating from any device with time-telling capability or illuminated screens (e.g., bedroom clock). As perceived stress may have implications on sleep duration and quality as well as daytime consequences of disturbed sleep [60], such devices should be eliminated. It also aims to reduce arousal during the night from unwanted cognitive activity [61], which may decrease sleep quality as described in the following section.

In summary, light strongly influences sleep. In this respect, sleep hygiene strategies that support the natural environmental light-dark cycle (i.e., red-light treatment prior to sleep, dawn-simulation therapy prior to waking) and prevent cycle disruption (i.e., filtering short wavelengths prior to sleep) should be recommended for elite soccer players.

\subsection{Relaxation and Brainwave Entrainment}

Elite soccer players may experience stress while trying to sleep in response to training, match, and/or lifestyle factors outside of sport. Excessive stress can cause severe emotional reactions that decrease sleep quality [62] and hinder the recovery process [63]. Stults-Kolehmainen et al. [63] demonstrated that high levels of stress (both life event stress and perceived stress) result in lower physical performance 4-day recovery from an acute heavy resistance exercise protocol. Consequently, under conditions of inordinate stress, the use of stress management techniques is highly desirable to enhance sleep and recovery.

Electroencephalogram-cortical oscillations can be divided into distinct bands (delta [1.5-6 Hz], theta [6.5-8 Hz], alpha $[8.5-12 \mathrm{~Hz}]$, beta $[8.5-30 \mathrm{~Hz}])$, and each specific frequency band is associated with different states of brain functioning [64]. Globally, stimulation of the midbrain reticular formation and other wake-active areas induces electroencephalogram activation and arousal (i.e., reduced theta activity, increased beta activity), whereas stimulation of the solitary tract nucleus, the ventrolateral preoptic area, and the median preoptic nucleus often induces electroencephalogram slowing and sleep (i.e., increased theta activity, reduced beta activity) $[27,64,65]$. When awake with eyes closed, the brainwave patterns of most individuals show unambiguous occipital alpha rhythm; the moment of sleep onset is associated with the abrupt disappearance of this alpha rhythm [33]. Almost without exception, the first sleep epoch is non-rapid-eye-movement stage 1 , characterized by increased theta activity and parasympathetic dominance [27, 33]. Consequently, any intervention that inhibit wake-promoting neurons or stimulate sleep-promoting neurons may facilitate the transition between arousal and sleep [27].

Brainwave entrainment and meditation are specific examples of this method of intervention. Abeln et al. [9] examined the effects of 8 weeks of brainwave entrainment on the sleep quality and post-sleep state of high-level soccer players. Brainwave entrainment involves stimulating the brain during sleep with frequencies between 1 and $9 \mathrm{~Hz}$ in a sequence mimicking the healthy human sleep cycle. Subjective ratings of sleep and awakening quality derived from the questionnaire of sleep and awakening quality [66], sleepiness and motivational state were significantly improved in the intervention group compared with the control group. Consequently, brainwave entrainment may be a promising sleep-promoting strategy for young sub-elite soccer players when applied chronically during periods of high physical and psychological loads and competitions. However, future studies are required to assess the effectiveness of brainwave entrainment among 
elite soccer players who face specific constraints (e.g., playing night matches with late kick-off, congested scheduling, travel). Further investigations should also include objective measurements, such as actigraphy or polysomnography, during sleep and performance tests to assess the effectiveness of the technique on sleep quality and quantity.

Electroencephalographic studies on meditation have demonstrated an overall electroencephalogram slowing (i.e., increased alpha and theta activity) [67, 68]. Consequently, meditation that is associated with relaxation and reduced stress [68] may be helpful for players with difficulty switching off the mind when attempting sleep [69, 70]. However, the applicability of meditation to elite soccer players is currently questionable and has yet to be shown. The large variety of techniques used [69] and/or the individual skill of the meditator [71] are potential biases to take into account in further research.

\subsection{Sleep Medication}

Sleep medication may be another option for artificially extending sleep duration. To prevent sleep loss and/or to counteract the effects of caffeine on sleep, athletes may take a hypnotic compound to get a 'good night's sleep' after competition. The available compounds known to improve sleep onset and/or sleep maintenance [72] include hypnotics, medium half-life benzodiazepines (e.g., temazepam) and short half-life non-benzodiazepine medications (e.g., zolpidem) [73, 74]; they do not contravene World Anti-Doping Agency rules. Mougin et al. [75] showed that delaying bedtime (i.e., the participants were not allowed to sleep before 3:00 am and were then allowed to sleep until 7:00 am) resulted in a decrease in the percentage of stages 1 and 2 and rapid-eye-movement sleep when compared with the reference night. However, administration of a hypnotic medication-induced sleep (10 mg zolpidem) did not result in a change in total sleep time or in any of the stages of sleep compared with the reference condition. According to these results, consuming hypnotic compounds seems neither beneficial nor detrimental to the outcome of sleep among well-trained male endurance athletes. Future studies are required to confirm these results when medication is consumed by soccer players after night matches; the survey on the sleep habits of professional soccer players revealed that $16 \%(N=3)$ of players take a hypnotic compound after night matches (unpublished observations). Additionally, the potential detrimental effects of sleeping medication must be recognized. Grobler et al. [76] found that subjects experience a significant hangover effect and altered eye-hand coordination and reaction time performance $10 \mathrm{~h}$ after sedative hypnotic administration. Mougin et al. [77] found that benzodiazepine-induced sleep led to sleep architecture modification among highly endurance trained subjects, i.e., an increase in stage 2 sleep, a decrease in wakefulness and in stage 3 sleep. Finally, benzodiazepines with established sleep maintenance efficacy are associated with next-day sedation and risk of tolerance and dependence [72].

Melatonin has been successfully used to help shift workers adjust to nocturnal regimens [78], to treat some sleeping disorders [78], and to reverse oxidative stress, improving total antioxidant status and immunological defenses among soccer players [79]. Melatonin can be naturally found in some foods (e.g., tart cherry juice) or it can be taken as a supplement. However, as the administration of exogenous melatonin may lead to hypnotic and hypothermic responses in humans and consequently reductions in short-term mental and physical performance for several hours after administration [78], future studies are required to justify melatonin supplement use among soccer players experiencing sleep disturbance. Furthermore, in addition to timing and dosage issues, concerns also exist as to its legal status in different countries and with the World Anti-Doping Agency [80].

\section{Sleep and Recovery Strategies Used in Soccer}

In elite soccer, different recovery strategies are combined in overall recovery plans [26]. Some strategies such as hydration, diet, cold water immersion, and sleep are effective in their ability to counteract the fatigue mechanisms [26]. Scientific evidence for active recovery, stretching, compression garments, massage, and electrical stimulation in their ability to accelerate the return to the initial level of performance is still lacking [26]. However, while it is important to isolate each recovery strategy to determine its effects on the recovery process, analyzing the potential interactions between recovery strategies is ecologically valid [81]. Consequently, the present section reviews the positive and/or negative interactions between sleep and recovery strategies currently used by elite soccer players.

\subsection{Sleep and Nutritional Strategies}

As previously reviewed [26], providing a high sodium drink (61 mmol/L) to players at a level equivalent to $150-200 \%$ of their sweat loss is sufficient to replenish water stores after match-induced dehydration $(\approx 2 \%$ of body mass) [82]; consumption of a chocolate milk drink at the end of competition can also notably reduce perceived soreness among players. Total fluid consumption after a match may have some negative effects on sleep (i.e., difficulty maintaining sleep, non-restorative sleep, and 
daytime sleepiness), due perhaps to fragmentation caused by more frequent sensations regarding urination [83]. Halson [84] reported that a major reason for sleep disturbances among elite athletes was waking several times during the night to urinate due to hyperhydration. In this respect, the intake of high-electrolyte fluids such as milk (133 $\mathrm{mg} \mathrm{Na}^{+}$and $431 \mathrm{mg} \mathrm{K}^{+}$in a $250 \mathrm{ml}$ serving [85]) should be preferred to low-sodium fluids (e.g., water) between cessation of match and bedtime in order to promote both fluid retention, rehydration, and potentially restorative sleep.

Serving a meal containing high-glycemic index carbohydrate $(\approx 1.2 \mathrm{~g}$ carbohydrate $/ \mathrm{kg} / \mathrm{h}$ ) and protein within the hour following a match is effective in replenishing substrate stores and optimizing muscle-damage repair [26]. However, post-match routines (e.g., medical care, recovery strategies, media demands, sponsor needs, and public interest [86]) may prevent such rapid post-match meal consumption, although, in any case one should also ask whether these nutritional recommendations are compatible with restorative sleep. Studies have suggested that particular foods/diets are important for re-entrainment of the peripheral circadian clock. For example, a meal high in carbohydrate, but low in protein may theoretically facilitate uptake of tryptophan and its conversion to serotonin, inducing drowsiness and sleep; on the other hand, a meal high in protein but low in carbohydrate might enhance tyrosine uptake and conversion to epinephrine, increasing arousal levels [28]. Studies investigating the effects of carbohydrate ingestion on indices of sleep quality and quantity are limited and provide contradictory results [11]. However, to counteract the potential decreased rate of muscle glycogen resynthesis among soccer players exposed to sleep deprivation [87], a preventive strategy may be to ensure additional carbohydrate is consumed in these circumstances [88]. Apart from quantity, the glycemic index of carbohydrate is also important to consider. Afaghi et al. [89] showed that a high-glycemic index meal resulted in a significant shortening of sleep-onset latency compared with a low-glycemic index meal (9.0 vs. $17.5 \mathrm{~min}$, respectively; $p<0.01$ ) and was most effective when consumed $4 \mathrm{~h}$ before bedtime compared with the same high-glycemic index meal given $1 \mathrm{~h}$ before bedtime.

Protein ingestion $(\approx 20 \mathrm{~g}$ milk protein or an equivalent of $\approx 9 \mathrm{~g}$ essential amino acids) after a night soccer match is required to optimize muscle-damage repair [26]. However, the timing and type of protein (i.e., containing more tryptophan than large neutral amino acids) must be carefully chosen, as it has been shown that protein consumption may have a significant influence on sleep [90, 91]. Schoenfeld et al. [92] conducted a multi-level meta-regression analysis of randomized controlled trials to determine whether protein timing is a viable strategy for enhancing post-exercise muscular adaptations. Results refute the commonly held belief that the timing of protein intake in and around ( $\leq 1 \mathrm{~h})$ a training session is critical to strength- or hypertrophic-related adaptations to resistance exercise [92]. Burk et al. [93] found significantly greater increases in strength and lean body mass from a time-divided protein dose (i.e., morning and evening) than the same dose provided around the resistance training session. However, protein ingestion immediately prior to sleep may provide an anabolic stimulus throughout the night. Res et al. [94] assessed the impact of ingestion of $40 \mathrm{~g}$ casein protein $30 \mathrm{~min}$ prior to sleep $(11: 30 \mathrm{pm})$ on digestion and absorption kinetics, whole-body protein balance, and mixed muscle protein synthesis rates throughout the overnight $(7.5 \mathrm{~h})$ recovery from a single bout of lower-limb resistance type exercise (eight sets of eight repetitions at 55-75\% 1-RM, both on the horizontal leg press machine and the leg extension machine) performed in the evening (8:00 pm). During sleep, protein was effectively digested and absorbed, resulting in a rapid rise in circulating amino acid levels that was sustained throughout the remainder of the night. Protein ingestion prior to sleep stimulated muscle protein synthesis rates by $\approx 22 \%$ and improved net protein balance $\quad\left(61 \pm 5\right.$ vs. $\quad-11 \pm 6 \mu \mathrm{mol} \mathrm{kg}{ }^{-1} \cdot 7.5 \mathrm{~h}^{-1}$, $p<0.01)$ compared with the placebo condition. Consequently, ingesting $40 \mathrm{~g}$ protein immediately prior to sleep may provide an additional anabolic stimulus to the coingestion of protein and carbohydrate immediately after exercise performed in the evening [95]. Future studies are required to confirm these results with soccer players experiencing sleep disturbance after a night soccer match. One practical application of these studies is providing a light bedtime snack or beverage (i.e., milk) to players, which may both provide an anabolic stimulus and positively influence sleep [21]. Other nutrients may enhance the recovery process of soccer players by influencing sleep quantity/quality. For example, tart cherry juice, which has been shown to enhance the recovery process [96, 97], may positively influence sleep. Howatson et al. [98] examined the effects of tart Montmorency cherry juice (Prunus cerasus) concentrate on the sleep of asymptomatic, healthy and young (26.6 \pm 4.6 years) individuals. Participants were instructed to consume two servings (after wakening and before the evening meal) of tart cherry juice concentrate (30 ml, corresponding to 90-100 tart cherries) each day for 7 days. Actigraphy results showed a significant increase in time in bed, total sleep time, and a 5-6\% increase in sleep efficiency in the cherry juice trial compared with both baseline and placebo trials. These results may be related to the total urinary 6-sulfatoxymelatonin content-the major metabolite of melatonin-that was significantly greater in the cherry juice trial than in baseline and placebo trials. Tart cherries also contain antioxidant 
and anti-inflammatory phytochemicals [97] that may influence sleep given that sleep regulation is also influenced by pro-inflammatory cytokines [99]. Melatonin is derived from tryptophan, which is an essential amino acid that can be absorbed exclusively from food in humans and that initiates the tryptophan-serotonin-melatonin pathway [100]. Doses of tryptophan as low as $1 \mathrm{~g}$ can improve sleep latency and subjective sleep quality; this can be achieved by consuming $\sim 300 \mathrm{~g}$ of turkey or $\sim 200 \mathrm{~g}$ of pumpkin seeds [101]. A significant sleep-inducing effect is observed after tryptophan administration during daytime or nighttime [102]. A combined tryptophan-rich breakfast (i.e., fermented soybeans and bananas) and evening-lighting have been shown effective to induce higher salivary melatonin secretion at night compared with control, which subjectively promoted onset of sleep and a higher quality of sleep among university soccer players [100]. These data suggest that consumption of a Montmorency tart cherry juice concentrate and/or tryptophan-rich breakfast provides an increase in exogenous melatonin that is beneficial in improving sleep duration and quality in healthy individuals and might therefore present a suitable adjunct intervention for disturbed sleep across a number of scenarios in healthy and symptomatic individuals. An investigation that examines acute and/or chronic consumption of tart cherry juice and/or tryptophan-rich breakfast concentrate among soccer players, perhaps with disturbed sleep related to matches, would be a valuable addition to the literature.

During sleep, one's posture should be supported to allow complete muscle relaxation and physical stillness [27]. However, during recovery from a soccer match, players are prone to delayed-onset muscle soreness [103, 104] that may induce difficulties remaining immobile during sleep and thus impairing sleep quantity and quality [105, 106]. Additionally, it has been shown that sleep restriction induces a significant increase of bodily discomfort, notably due to significant increases of generalized body pain and back pain [107]. Muscle soreness is likely part of a cycle of poor sleep leading to increased muscle soreness, which in turn promotes impaired sleep. Interestingly, some authors have suggested a link between abnormally low levels of vitamin D (25-hydroxyvitamin $\mathrm{D}<20 \mathrm{ng} / \mathrm{ml}$ ) and difficulty maintaining asleep [83, 106]. Vitamin D can be naturally found in some foods (e.g., kippers, mackerel) or it can be taken as a supplement. Vitamin D deficiency may be a cause of or a contributor to common sleep-disorder symptoms, including chronic nonspecific musculoskeletal soreness, making it difficult to remain immobile during sleep and thus impairing sleep efficiency [106]. As soccer players are at risk of vitamin D deficiency [108], soccer medical staff should be familiar with the scope of disease potentially caused by inadequacy of vitamin $\mathrm{D}$, and should be vigilant regarding detection and proper treatment. Future studies are required to ascertain the potential detrimental effect of muscle soreness on subsequent sleep among soccer players. When experiencing delayed onset muscle soreness, it may be advisable to recommend players use recovery strategies aimed at reducing soreness (e.g., cold water immersion, contrast water therapy, compression garments, massage) in an attempt to indirectly improve sleep quantity and quality.

\subsection{Sleep, Cold Water Immersion and Passive Distal Heating}

Recovery strategies aimed at reducing acute inflammation from muscle damage are particularly common in professional soccer settings. Cold water immersion (temperature range: $9-10{ }^{\circ} \mathrm{C}$; duration range: $10-20 \mathrm{~min}$ ) immediately after competition is an effective recovery strategy during acute periods of fixture congestion to improve physical performance, reduce muscle soreness, and repress the acute inflammatory process [26]. The present section reviews the compatibility between cold water immersion and restorative sleep.

The recovery benefits of cold water immersion are most likely due to the combination of water temperature and hydrostatic pressure [109, 110]. Cold water immersion rapidly decreases core temperature to below normal levels for up to $90 \mathrm{~min}$ post-immersion [111, 112]. If performed close to bedtime, cold water immersion-induced changes in core temperature could potentially affect sleep by accelerating the normal pre-sleep rate of decline of core temperature and improve sleep propensity [113]. Several studies have been conducted to assess the effect of cold water immersion after exercise on subsequent sleep quantity and quality [114-116]. Al Haddad et al. [114] have shown a positive effect of daily cold $\left(5 \mathrm{~min}\right.$ at $\left.15^{\circ} \mathrm{C}\right)$ water immersion throughout 5 consecutive training days on rating of perceived sleep quality in swimmers, but positive effects on objective sleep measures are lacking [115, 116]. Conversely, Halson et al. [117] compared the effects of cold water immersion (four sessions per week; $15 \mathrm{~min}$ at $\approx 15^{\circ} \mathrm{C}$ ) and passive rest during a 39-day intensified cycling training period and demonstrated that, after 1 week of taper following the intensified training period, cold water immersion had a possibly harmful effect $(2 \mathrm{~min})$ on total sleep time and a moderately harmful effect $(5 \mathrm{~min})$ on sleep latency compared with control. In these studies, the overall absence of positive effect on objective sleep parameters may be explained by the fact that early-evening exercise (between 4:30 and 7:00 pm) was performed. 
Consequently, cold water immersion-induced changes in core temperature may not have been persistent up to bedtime. Future studies are required to assess the potential impact of cold water immersion on sleep after soccer matches performed later in the night (e.g., 9:00 pm). However, as post-match routines (i.e., medical care, recovery strategies, meals, and the return travel) frequently lead to a very late bedtime [26], the time period between cold water immersion and bedtime should be carefully considered. Future studies should also consider the potential interest in simultaneously cooling the core and warming the skin, as Van Someren et al. [118] found that drinking an iced beverage-which mildly cools the body core-and wearing a heated suit at the same time shortened sleep-onset latency more than any other combination of a warm or cold beverage with a warm or cold suit [118]. According to Kräuchi [113], body heat loss before lights off, via increased skin temperature and selective vasodilatation of distal regions (i.e., hands and feet), promotes sleepiness and the rapid onset of sleep. Accordingly, it has been shown that sleep onset may be accelerated by a warm footbath prior to lights off and/or by 'normal' bed socks applied after lights off [119]. Alternatively, compression garments, which are integrated into the recovery protocol of $22 \%$ of French professional soccer teams, may be uncomfortable to wear during the night and are associated with an increase in body temperature, thereby disturbing subjects' patterns of sleep [120]. Consequently, future studies are required to determine the potential interest in heating of passive distal regions on sleep parameters among soccer players.

Alternatively, cold water immersion performed close to bedtime may negatively affect sleep. It has been shown that cold water immersion $\left(15 \mathrm{~min}\right.$ at $\left.15{ }^{\circ} \mathrm{C}\right)$ significantly increases electrocortical beta3 activity [64]. Since electrocortical reduced theta activity and increased beta activity characterize electroencephalogram activation and arousal [27, 64, 65], a cold water immersion-induced electrocortical state may not favor sleep induction. Additionally, Harris [121] has previously described the physiological changes during the transition from wakefulness to sleep, which includes a decrease in sympathetic activity and an increase in parasympathetic activity. Cold water immersion increases the activity of the sympathetic nervous system and arousal, as shown by the increase in plasma noradrenaline and dopamine concentrations [122].

\subsection{Sleep and Other Recovery Strategies}

Scientific evidence for the efficacy of active recovery, stretching, compression garments, massage, and electrical stimulation to accelerate a return to the initial level of performance is lacking [26]. Consequently, it is deemed crucial that the use of these recovery strategies does not negatively affect sleep. Conversely, even if active recovery, stretching, compression garments, massage, or electrical stimulation do not significantly accelerate the return to initial levels of performance in comparison with a control condition, such strategies may positively affect sleep and promote the overall recovery outcome. To our knowledge, only Duffield et al. [123] have examined the effects of combining recovery strategies (i.e., $15 \mathrm{~min}$ cold water immersion, $3 \mathrm{~h}$ of wearing full-body compression garments, sleep hygiene recommendations) on the recovery process and sleep quantity and quality following twice daily on-court tennis training and match-play sessions. The sleep hygiene recommendations involved participants creating a low-light $(8 \pm 5$ lux $)$ and cool $\left(19 \pm 2{ }^{\circ} \mathrm{C}\right)$ environment at 9:00 pm. Specifically, participants ensured all electronic stimulants, i.e., television, mobile phones, and computers, were limited or avoided and excessive light was minimized during the ensuing $30 \mathrm{~min}$ until 9:30 pm, by which time they had retired to bed wearing eye masks. Conversely, in the control condition, players were not provided with cold water immersion or compression and were allowed to self-regulate exposure to electronic equipment, pre-bed light (60 \pm 12 lux), and sleeping patterns. Large effects for increased sleep duration in the recovery condition were evident with the implementation of the sleep hygiene recommendations. The large effects observed for increased sleep duration resulted from both an increased total time in bed and minutes asleep. Perceived muscle soreness remained lower until the next morning following compression garments and sleep interventions compared with control. Large effect sizes were evident for reduced feelings of fatigue the following morning after the sleep hygiene recommendations. Consequently, current data suggest that players should be educated on the appropriate activities, behaviors, and sleep hygiene recommendations to follow post match [124] that may improve acute sleep quantity in athletes and may additionally assist with improving perceptual recovery of soreness and mood the following day [123]. As the vulnerability to developing sleep disturbance seems to differ between individuals $[15,22]$, elaborating customized sleep hygiene strategies from a set of sleep hygiene recommendations may also be recommended.

\section{Conclusion}

The constraints of elite soccer may influence a player's ability to sleep adequately, potentially leading to sleep deprivation. Assuming that the optimal way to treat a 
Table 1 Potential sleep hygiene strategies for promoting sleep quantity and/or quality after night soccer match

Use glasses fitted with short-wavelength filters after the match

(e.g., in the airport, travelling from airport) [51]

Consume high-electrolyte fluids such as milk rather than lowsodium fluids (e.g., water) between cessation of match and bedtime [82, 85]

Consume a high glycemic index meal [89]

Consume protein immediately prior to sleep (e.g., milk) [94]

Consume Montmorency tart cherry juice concentrate and/or tryptophan-containing foods (e.g., turkey, pumpkin seeds) [98, 100]

Use recovery strategies aimed at reducing muscle soreness (e.g., cold water immersion, contrast water therapy, compression garments, massage)

Create a low-light and cool $\left(18-19^{\circ} \mathrm{C}\right)$ sleep environment, avoid all electronic stimulants (i.e., television, mobile phones, computers) in the hour prior to sleep [123, 125]

Eliminate the bedroom clock [61]

Explore the use of brainwave entrainment and meditation [9, 69]

Use acute dawn-simulation therapy during the last $30 \mathrm{~min}$ prior to waking (especially for late chronotypes) [46]

Have a regular bedtime/wake time and avoid sleeping too late in the morning off days [21, 34]

Nap briefly (i.e., 5-30 min) and appropriately (close to the early afternoon and not during the morning or evening) [37, 38, 40]

Engage in active daytime behaviors (e.g., light exercise) and bright morning light exposure during off days [42, 43]

constraint is to match the treatment with the specific symptoms, the present article identified conducive sleep hygiene and recovery strategies to promote restorative sleep (Table 1). Sleep extension is a suitable strategy to counteract sleep deprivation, but sleep hygiene strategies are required to promote restorative sleep. Sleep hygiene strategies that support the natural environmental light-dark cycle and prevent cycle disruption are available to manage the impact of light on sleep. Brainwave entrainment and meditation may be helpful techniques for players experiencing high levels of stress. In elite soccer, several recovery strategies are combined in overall recovery plans, and future studies are required to establish the interactions between sleep and recovery strategies. A synergy between strategies is still to be found in order to positively affect sleep and promote the overall recovery outcome. However, the difficulty of attaining such synergy in field-based environments is recognized. Sleep initiation is influenced by numerous factors, and appropriate sleep hygiene strategies that players might self-administer post match and throughout the training week are required. A focus on customized sleep hygiene strategies is needed.

Acknowledgments No sources of funding were used to assist in the preparation of this review. The authors have no conflicts of interest that are directly relevant to the content of this review.

\section{References}

1. Nédélec M, McCall A, Carling C, et al. Recovery in soccer: part I-post-match fatigue and time course of recovery. Sports Med. 2012;42(12):997-1015.

2. Akerstedt T, Nilsson PM. Sleep as restitution: an introduction. J Intern Med. 2003;254(1):6-12.

3. Walters PH. Sleep, the athlete, and performance. Strength Cond J. 2002;24:17-24.

4. Diekelmann S, Born J. The memory function of sleep. Nat Rev Neurosci. 2010;11(2):114-26.

5. Maquet P, Laureys S, Peigneux P, et al. Experience-dependent changes in cerebral activation during human REM sleep. Nat Neurosci. 2000;3(8):831-6.

6. Peigneux P, Laureys S, Fuchs S, et al. Are spatial memories strengthened in the human hippocampus during slow wave sleep? Neuron. 2004;44(3):535-45.

7. Nishida M, Walker MP. Daytime naps, motor memory consolidation and regionally specific sleep spindles. PLoS One. 2007;2(4):e341.

8. Frank MG. The mystery of sleep function: current perspectives and future directions. Rev Neurosci. 2006;17(4):375-92.

9. Abeln V, Kleinert J, Strüder HK, et al. Brainwave entrainment for better sleep and post-sleep state of young elite soccer players-a pilot study. Eur J Sport Sci. 2014;14(5):393-402.

10. Barbato G, Barker C, Bender C, et al. Extended sleep in humans in 14 hour nights (LD 10:14): relationship between REM density and spontaneous awakening. Electroencephalogr Clin Neurophysiol. 1994;90(4):291-7.

11. Halson SL. Sleep in elite athletes and nutritional interventions to enhance sleep. Sports Med. 2014;44(Suppl 1):S13-23.

12. Kamdar BB, Kaplan KA, Kezirian EJ, et al. The impact of extended sleep on daytime alertness, vigilance, and mood. Sleep Med. 2004;5(5):441-8.

13. Mah CD, Mah KE, Dement WC. Extended sleep and the effects on mood and athletic performance in collegiate swimmers. Sleep. 2008;31:A128 (abstract supplement).

14. Mah CD, Mah KE, Kezirian EJ, et al. The effects of sleep extension on the athletic performance of collegiate basketball players. Sleep. 2011;34(7):943-50.

15. Leeder J, Glaister M, Pizzoferro K, et al. Sleep duration and quality in elite athletes measured using wristwatch actigraphy. J Sports Sci. 2012;30(6):541-5.

16. Natale V, Plazzi G, Martoni M. Actigraphy in the assessment of insomnia: a quantitative approach. Sleep. 2009;32(6):767-71.

17. Fullagar HHK. Late-night matches for professional soccer players: should we be concerned about sleep and recovery? Int J Sports Exerc Med. 2015;1:002e.

18. Meyer T, Wegmann M, Poppendieck W, et al. Regenerative interventions in professional football. Sport Orthop Traumatol. 2014;30:112-8

19. Association American Sleep Disorders. The international classification of sleep disorders, revised: diagnostic and coding manual. Rochester: American Sleep Disorders Association; 1997.

20. Fullagar HH, Skorski S, Duffield R, et al. Sleep and athletic performance: the effects of sleep loss on exercise performance, and physiological and cognitive responses to exercise. Sports Med. 2015;45(2):161-86.

21. Hauri P. Current concepts: the sleep disorders. Kalamazoo: The Upjohn Company; 1977.

22. Stepanski EJ, Wyatt JK. Use of sleep hygiene in the treatment of insomnia. Sleep Med Rev. 2003;7(3):215-25.

23. Drake C, Roehrs T, Shambroom J, et al. Caffeine effects on sleep taken 0,3 , or 6 hours before going to bed. J Clin Sleep Med. 2013;9(11):1195-200. 
24. Feige B, Gann H, Brueck R, et al. Effects of alcohol on polysomnographically recorded sleep in healthy subjects. Alcohol Clin Exp Res. 2006;30(9):1527-37.

25. Kredlow MA, Capozzoli MC, Hearon BA, et al. The effects of physical activity on sleep: a meta-analytic review. J Behav Med. 2015;38(3):427-49.

26. Nédélec M, McCall A, Carling C, et al. Recovery in soccer: part II-recovery strategies. Sports Med. 2013;43(1):9-22.

27. Cole RJ. Nonpharmacologic techniques for promoting sleep. Clin Sports Med. 2005;24(2):343-53 (xi).

28. Leatherwood WE, Dragoo JL. Effect of airline travel on performance: a review of the literature. $\mathrm{Br} \mathrm{J}$ Sports Med. 2013;47(9):561-7.

29. Sargent C, Schmidt WF, Aughey RJ, et al. The impact of altitude on the sleep of young elite soccer players (ISA3600). Br J Sports Med. 2013;47(Suppl 1):i86-92.

30. Roky R, Herrera CP, Ahmed Q. Sleep in athletes and the effects of Ramadan. J Sports Sci. 2012;30(Suppl 1):S75-84.

31. Erlacher D, Ehrlenspiel F, Adegbesan OA, et al. Sleep habits in German athletes before important competitions or games. J Sports Sci. 2011;29(8):859-66.

32. Emsellem HA, Murtagh KE. Sleep apnea and sports performance. Clin Sports Med. 2005;24(2):329-41 (x).

33. Dement WC. Sleep extension: getting as much extra sleep as possible. Clin Sports Med. 2005;24(2):251-68 (viii).

34. Kabrita CS, Hajjar-Muça TA, Duffy JF. Predictors of poor sleep quality among Lebanese university students: association between evening typology, lifestyle behaviors, and sleep habits. Nat Sci Sleep. 2014;6:11-8.

35. Spielman AJ, Saskin P, Thorpy MJ. Treatment of chronic insomnia by restriction of time in bed. Sleep. 1987;10(1):45-56.

36. Lastella M, Roach GD, Halson SL, et al. Sleep/wake behaviours of elite athletes from individual and team sports. Eur J Sport Sci. 2015;15(2):94-100.

37. Takahashi M. The role of prescribed napping in sleep medicine. Sleep Med Rev. 2003;7(3):227-35.

38. Dhand R, Sohal H. Good sleep, bad sleep! The role of daytime naps in healthy adults. Curr Opin Pulm Med. 2006;12(6):379-82.

39. Lovato N, Lack L. The effects of napping on cognitive functioning. Prog Brain Res. 2010;185:155-66.

40. Lavie P. Ultrashort sleep-waking schedule. III. 'Gates' and 'forbidden zones' for sleep. Electroencephalogr Clin Neurophysiol. 1986;63(5):414-25.

41. Lavie P, Zvuluni A. The 24-hour sleep propensity function: experimental bases for somnotypology. Psychophysiology. 1992;29(5):566-75.

42. Youngstedt SD. Effects of exercise on sleep. Clin Sports Med. 2005;24(2):355-65 (xi).

43. Campbell SS, Dawson D, Anderson MW. Alleviation of sleep maintenance insomnia with timed exposure to bright light. J Am Geriatr Soc. 1993;41(8):829-36.

44. Cajochen C. Alerting effects of light. Sleep Med Rev. 2007;11(6):453-64.

45. Shochat T. Impact of lifestyle and technology developments on sleep. Nat Sci Sleep. 2012;4:19-31.

46. Thompson A, Jones H, Gregson W, et al. Effects of dawn simulation on markers of sleep inertia and post-waking performance in humans. Eur J Appl Physiol. 2014;114(5):1049-56.

47. Radogna F, Diederich M, Ghibelli L. Melatonin: a pleiotropic molecule regulating inflammation. Biochem Pharmacol. 2010;80(12):1844-52.

48. Wright HR, Lack LC. Effect of light wavelength on suppression and phase delay of the melatonin rhythm. Chronobiol Int. 2001;18(5):801-8.
49. Figueiro MG, Rea MS. The effects of red and blue lights on circadian variations in cortisol, alpha amylase, and melatonin. Int J Endocrinol. 2010;2010:829351.

50. Zee PC, Attarian H, Videnovic A. Circadian rhythm abnormalities. Continuum (Minneap Minn). 2013;19(1 Sleep Disorders):132-47.

51. Rahman SA, Shapiro CM, Wang F, et al. Effects of filtering visual short wavelengths during nocturnal shiftwork on sleep and performance. Chronobiol Int. 2013;30(8):951-62.

52. Zhao J, Tian Y, Nie J, et al. Red light and the sleep quality and endurance performance of Chinese female basketball players. J Athl Train. 2012;47(6):673-8.

53. Buysse DJ, Reynolds CF 3rd, Monk TH, et al. The Pittsburgh Sleep Quality Index: a new instrument for psychiatric practice and research. Psychiatry Res. 1989;28(2):193-213.

54. Jewett ME, Wyatt JK, Ritz-De Cecco A, et al. Time course of sleep inertia dissipation in human performance and alertness. J Sleep Res. 1999;8(1):1-8.

55. Horne JA, Ostberg O. A self-assessment questionnaire to determine morningness-eveningness in human circadian rhythms. Int J Chronobiol. 1976;4(2):97-110.

56. Roenneberg T, Wirz-Justice A, Merrow M. Life between clocks: daily temporal patterns of human chronotypes. J Biol Rhythms. 2003;18(1):80-90.

57. Cajochen C, Kräuchi K, Wirz-Justice A. Role of melatonin in the regulation of human circadian rhythms and sleep. J Neuroendocrinol. 2003;15(4):432-7.

58. Gabel V, Maire M, Reichert CF, et al. Effects of artificial dawn and morning blue light on daytime cognitive performance, wellbeing, cortisol and melatonin levels. Chronobiol Int. 2013;30(8):988-97.

59. Postolache TT, Hung TM, Rosenthal RN, et al. Sports chronobiology consultation: from the lab to the arena. Clin Sports Med. 2005;24(2):415-56 (xiv).

60. Kashani M, Eliasson A, Vernalis M. Perceived stress correlates with disturbed sleep: a link connecting stress and cardiovascular disease. Stress. 2012;15(1):45-51.

61. Hauri P. Sleep hygiene, relaxation therapy, and cognitive interventions. In: Hauri PJ, editor. Case studies in insomnia. New York: Plenum; 1992. p. 65-84.

62. Silva A, Queiroz SS, Winckler C, et al. Sleep quality evaluation, chronotype, sleepiness and anxiety of Paralympic Brazilian athletes: Beijing 2008 Paralympic Games. Br J Sports Med. 2012;46(2):150-4.

63. Stults-Kolehmainen MA, Bartholomew JB, Sinha R. Chronic psychological stress impairs recovery of muscular function and somatic sensations over a 96-hour period. J Strength Cond Res. 2014;28(7):2007-17.

64. De Pauw K, Roelands B, Marusic U, et al. Brain mapping after prolonged cycling and during recovery in the heat. J Appl Physiol (1985). 2013;115(9):1324-31.

65. Moraes H, Ferreira C, Deslandes A, et al. Beta and alpha electroencephalographic activity changes after acute exercise. Arq Neuropsiquiatr. 2007;65(3A):637-41.

66. Saletu B, Wessely $P$, Grünberger $J$, et al. Erste klinische Erfahrungen mit einem neuen schlafanstoßenden Benzodiazepin, Cinolazepam, mittels eines Selbstbeurteilungsbogens für Schlafund Aufwachqualität (SSA). Neuropsychiatrie. 1987;1(4):169-76.

67. Cahn BR, Polich J. Meditation states and traits: EEG, ERP, and neuroimaging studies. Psychol Bull. 2006;132(2):180-211.

68. Chiesa A. Zen meditation: an integration of current evidence. J Altern Complement Med. 2009;15(5):585-92.

69. Kozasa EH, Hachul H, Monson C, et al. Mind-body interventions for the treatment of insomnia: a review. Rev Bras Psiquiatr. 2010;32(4):437-43. 
70. Ong JC, Manber R, Segal Z, et al. A randomized controlled trial of mindfulness meditation for chronic insomnia. Sleep. 2014;37(9):1553-63.

71. Debarnot U, Sperduti M, Di Rienzo F, et al. Experts bodies, experts minds: How physical and mental training shape the brain. Front Hum Neurosci. 2014;8:280.

72. Rosenberg RP. Sleep maintenance insomnia: strengths and weaknesses of current pharmacologic therapies. Ann Clin Psychiatry. 2006;18(1):49-56.

73. Gremion G, Sutter-Weyrich C, Rostan A, et al. Physical performance and sedation: comparative study of the effects of a benzodiazepine (temazepam) and of a non-benzodiazepine hypnotic (zolpidem) [Article in French]. Schweiz Z Sportmed. 1992;40(3):113-8.

74. Lagarde D. Pharmacological approach to desychronization of the sleep-wakefulness cycle in the military and sport environment. [Article in French]. Ann Pharm Fr. 2007;65(4):258-64.

75. Mougin F, Bourdin H, Simon-Rigaud ML, et al. Hormonal responses to exercise after partial sleep deprivation and after a hypnotic drug-induced sleep. J Sports Sci. 2001;19(2):89-97.

76. Grobler LA, Schwellnus MP, Trichard C, et al. Comparative effects of zopiclone and loprazolam on psychomotor and physical performance in active individuals. Clin J Sport Med. 2000;10(2):123-8.

77. Mougin F, Simon-Rigaud ML, Davenne D, et al. Effects of sleep disturbances on subsequent physical performance. Eur J Appl Physiol Occup Physiol. 1991;63(2):77-82.

78. Atkinson G, Drust B, Reilly T, et al. The relevance of melatonin to sports medicine and science. Sports Med. 2003;33(11):809-31.

79. Maldonado MD, Manfredi M, Ribas-Serna J, et al. Melatonin administrated immediately before an intense exercise reverses oxidative stress, improves immunological defenses and lipid metabolism in football players. Physiol Behav. 2012;105(5):1099-103.

80. Forbes-Robertson S, Dudley E, Vadgama P, et al. Circadian disruption and remedial interventions: effects and interventions for jet lag for athletic peak performance. Sports Med. 2012;42(3):185-208.

81. Lovell R, Midgley A, Barrett S, et al. Effects of different halftime strategies on second half soccer-specific speed, power and dynamic strength. Scand J Med Sci Sports. 2013;23(1):105-13.

82. Shirreffs SM, Taylor AJ, Leiper JB, et al. Post-exercise rehydration in man: effects of volume consumed and drink sodium content. Med Sci Sports Exerc. 1996;28(10):1260-71.

83. Grandner MA, Jackson N, Gerstner JR, et al. Sleep symptoms associated with intake of specific dietary nutrients. J Sleep Res. 2014;23(1):22-34

84. Halson SL. Nutrition, sleep and recovery. Eur J Sport Sci. 2008;8(2):119-26.

85. Roy BD. Milk: the new sports drink? A review. J Int Soc Sports Nutr. 2008;5:15.

86. Botterill C, Wilson C. Overtraining: emotional and interdisciplinary dimensions. In: Kellmann M, editor. Enhancing recovery: preventing underperformance in athletes. Champaign: Human Kinetics; 2002. p. 143-59.

87. Skein M, Duffield R, Edge J, et al. Intermittent-sprint performance and muscle glycogen after $30 \mathrm{~h}$ of sleep deprivation. Med Sci Sports Exerc. 2011;43(7):1301-11.

88. Burke LM, Loucks AB, Broad N. Energy and carbohydrate for training and recovery. J Sports Sci. 2006;24(7):675-85.

89. Afaghi A, O'Connor H, Chow CM. High-glycemic-index carbohydrate meals shorten sleep onset. Am J Clin Nutr. 2007;85(2):426-30.

90. Lacey JH, Hawkins C, Crisp AH. Effects of dietary protein on sleep E.E.G. in normal subjects. Adv Biosci. 1978;21:245-7.
91. Lindseth G, Lindseth P, Thompson M. Nutritional effects on sleep. West J Nurs Res. 2013;35(4):497-513.

92. Schoenfeld BJ, Aragon AA, Krieger JW. The effect of protein timing on muscle strength and hypertrophy: a meta-analysis. J Int Soc Sports Nutr. 2013;10(1):53.

93. Burk A, Timpmann S, Medijainen L, et al. Time-divided ingestion pattern of casein-based protein supplement stimulates an increase in fat-free body mass during resistance training in young untrained men. Nutr Res. 2009;29(6):405-13.

94. Res PT, Groen B, Pennings B, et al. Protein ingestion before sleep improves postexercise overnight recovery. Med Sci Sports Exerc. 2012;44(8):1560-9.

95. Beelen M, Tieland M, Gijsen AP, et al. Coingestion of carbohydrate and protein hydrolysate stimulates muscle protein synthesis during exercise in young men, with no further increase during subsequent overnight recovery. $J$ Nutr. 2008;138(11):2198-204.

96. Connolly DA, McHugh MP, Padilla-Zakour OI, et al. Efficacy of a tart cherry juice blend in preventing the symptoms of muscle damage. Br J Sports Med. 2006;40(8):679-83 (discussion 683).

97. Howatson G, McHugh MP, Hill JA, et al. Influence of tart cherry juice on indices of recovery following marathon running. Scand J Med Sci Sports. 2010;20(6):843-52.

98. Howatson G, Bell PG, Tallent J, et al. Effect of tart cherry juice (Prunus cerasus) on melatonin levels and enhanced sleep quality. Eur J Nutr. 2012;51(8):909-16.

99. Opp MR. Cytokines and sleep: the first hundred years. Brain Behav Immun. 2004;18(4):295-7.

100. Wada K, Yata S, Akimitsu O, et al. A tryptophan-rich breakfast and exposure to light with low color temperature at night improve sleep and salivary melatonin level in Japanese students. J Circadian Rhythms. 2013;11(1):4.

101. Halson SL. Nutritional interventions to enhance sleep. Sports Science Exchange. 2013;26(116):1-5.

102. Hajak G, Huether G, Blanke J, et al. The influence of intravenous L-tryptophan on plasma melatonin and sleep in men. Pharmacopsychiatry. 1991;24(1):17-20.

103. Fatouros IG, Chatzinikolaou A, Douroudos II, et al. Time-course of changes in oxidative stress and antioxidant status responses following a soccer game. J Strength Cond Res. 2010;24(12):3278-86.

104. Ispirlidis I, Fatouros IG, Jamurtas AZ, et al. Time-course of changes in inflammatory and performance responses following a soccer game. Clin J Sport Med. 2008;18(5):423-31.

105. Hausswirth C, Louis J, Aubry A, et al. Evidence of disturbed sleep and increased illness in overreached endurance athletes. Med Sci Sports Exerc. 2014;46(5):1036-45.

106. McCarty DE, Chesson AL Jr, Jain SK, et al. The link between vitamin D metabolism and sleep medicine. Sleep Med Rev. 2014;18(4):311-9.

107. Haack M, Mullington JM. Sustained sleep restriction reduces emotional and physical well-being. Pain. 2005;119(1-3):56-64.

108. Hamilton B, Whiteley R, Farooq A, et al. Vitamin D concentration in 342 professional football players and association with lower limb isokinetic function. J Sci Med Sport. 2014;17(1):139-43.

109. Ingram J, Dawson B, Goodman C, et al. Effect of water immersion methods on post-exercise recovery from simulated team sport exercise. J Sci Med Sport. 2009;12(3):417-21.

110. Rowsell GJ, Coutts AJ, Reaburn P, et al. Effect of post-match cold-water immersion on subsequent match running performance in junior soccer players during tournament play. J Sports Sci. 2011;29(1):1-6.

111. Gregson W, Black MA, Jones H, et al. Influence of cold water immersion on limb and cutaneous blood flow at rest. Am J Sports Med. 2011;39(6):1316-23. 
112. Robey E, Dawson B, Halson S, et al. Post-exercise cold water immersion: effect on core temperature and melatonin responses. Eur J Appl Physiol. 2013;113(2):305-11.

113. Kräuchi K. The thermophysiological cascade leading to sleep initiation in relation to phase of entrainment. Sleep Med Rev. 2007;11(6):439-51.

114. Al Haddad H, Parouty J, Buchheit M. Effect of daily cold water immersion on heart rate variability and subjective ratings of well-being in highly trained swimmers. Int J Sports Physiol Perform. 2012;7(1):33-8.

115. Robey E, Dawson B, Halson S, et al. Effect of evening postexercise cold water immersion on subsequent sleep. Med Sci Sports Exerc. 2013;45(7):1394-402.

116. Robey E, Dawson B, Halson S, et al. Sleep quantity and quality in elite youth soccer players: a pilot study. Eur J Sport Sci. 2014;14(5):410-7.

117. Halson SL, Bartram J, West N, et al. Does hydrotherapy help or hinder adaptation to training in competitive cyclists? Med Sci Sports Exerc. 2014;46(8):1631-9.

118. Van Someren EJ, Raymann R, Drosopoulos S, et al. Effect of body temperature manipulation on pulse wave amplitude and sleep onset latency. Sleep. 2002;25(Abstract Suppl):A128.
119. Raymann RJ, Swaab DF, Van Someren EJ. Skin temperature and sleep-onset latency: changes with age and insomnia. Physiol Behav. 2007;90(2-3):257-66.

120. Davies V, Thompson KG, Cooper SM. The effects of compression garments on recovery. J Strength Cond Res. 2009;23(6):1786-94.

121. Harris CD. Neurophysiology of sleep and wakefulness. Respir Care Clin N Am. 2005;11(4):567-86.

122. Srámek P, Simecková M, Janský L, et al. Human physiological responses to immersion into water of different temperatures. Eur J Appl Physiol. 2000;81(5):436-42.

123. Duffield R, Murphy A, Kellett A, et al. Recovery from repeated on-court tennis sessions: combining cold-water immersion, compression, and sleep recovery interventions. Int J Sports Physiol Perform. 2014;9(2):273-82.

124. Prentice C, Stannard SR, Barnes MJ. The effects of binge drinking behaviour on recovery and performance after a rugby match. J Sci Med Sport. 2014;17(2):244-8.

125. Bird SP. Sleep, recovery, and athletic performance: a brief review and recommendations. Strength Cond J. 2013;35(5):43-7. 